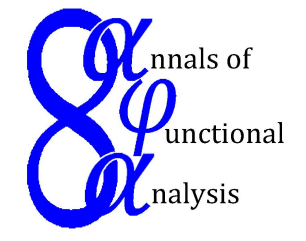

Ann. Funct. Anal. 5 (2014), no. 1, 30-35

$\mathscr{A}$ NNALS OF $\mathscr{F}$ UNCTIONAL $\mathscr{A}$ NALYSIS

ISSN: 2008-8752 (electronic)

URL:www.emis.de/journals/AFA/

\title{
ZERO-DILATION INDICES OF KMS MATRICES
}

\author{
HWA-LONG GAU ${ }^{1}$ AND PEI YUAN WU ${ }^{2 *}$ \\ Dedicated to Professor Tsuyoshi Ando with admiration \\ Communicated by Q.-W. Wang
}

\begin{abstract}
The zero-dilation index $d(A)$ of an $n$-by- $n$ complex matrix $A$ is the maximum size of the zero matrix which can be dilated to $A$. In this paper, we determine the value of this index for the KMS matrix
\end{abstract}

$$
J_{n}(a)=\left[\begin{array}{ccccc}
0 & a & a^{2} & \cdots & a^{n-1} \\
& 0 & a & \ddots & \vdots \\
& & \ddots & \ddots & a^{2} \\
& & & \ddots & a \\
0 & & & & 0
\end{array}\right], a \in \mathbb{C} \text { and } n \geq 1,
$$

by using the Li-Sze characterization of higher-rank numerical ranges of a finite matrix.

\section{INTRODUCTION AND PRELIMINARIES}

For any $n$-by- $n$ complex matrix $A$, let $d(A)$ denote the maximum size of a zero matrix which can be dilated to $A$, called the zero-dilation index of $A$. Recall that a $k$-by-k matrix $B$ is said to dilate to $A$ if $B=V^{*} A V$ for some $n$-by- $k$ matrix $V$ with $V^{*} V=I_{k}$, the $k$-by- $k$ identity matrix, or, equivalently, if $A$ is unitarily similar to a matrix of the form $\left[\begin{array}{cc}B & * \\ * & *\end{array}\right]$. Hence the zero-dilation index of $A$ can

Date: Received: 2 April 2013; Accepted: 3 June 2013.

* Corresponding author.

2010 Mathematics Subject Classification. Primary 47A20; Secondary 15B05, 15A60.

Key words and phrases. Zero-dilation index, KMS matrix, higher-rank numerical range, $S_{n^{-}}$ matrix, $S_{n}^{-1}$-matrix. 
also be expressed as

$$
d(A)=\max \left\{k \geq 1: A \text { is unitarily similar to }\left[\begin{array}{cc}
0_{k} & * \\
* & *
\end{array}\right]\right\}
$$

where $0_{k}$ denotes the $k$-by- $k$ zero matrix. The study of $d(A)$ was initiated in [4], in which we established its basic properties and its relations with the eigenvalues of $A$, and we determined the value of $d(A)$ when $A$ is a normal matrix or a weighted permutation matrix with zero diagonals. The main tool we used there is the Li-Sze characterization of higher-rank numerical ranges of $A$. Recall that for any integer $k, 1 \leq k \leq n$, the rank- $k$ numerical range $\Lambda_{k}(A)$ of $A$ is the subset $\left\{\lambda \in \mathbb{C}: \lambda I_{k}\right.$ dilates to $\left.A\right\}$ of the complex plane. Note that $\Lambda_{1}(A)$ coincides with the classical numerical range $W(A)=\left\{\langle A x, x\rangle: x \in \mathbb{C}^{n},\|x\|=1\right\}$ of $A$, where $\langle\cdot, \cdot\rangle$ and $\|\cdot\|$ are the standard inner product and its associated norm in $\mathbb{C}^{n}$. Li and Sze gave in [9, Theorem 2.2] a specific description of $\Lambda_{k}(A)$, namely,

$$
\Lambda_{k}(A)=\bigcap_{\theta \in \mathbb{R}}\left\{\lambda \in \mathbb{C}: \operatorname{Re}\left(e^{-i \theta} \lambda\right) \leq \lambda_{k}\left(\operatorname{Re}\left(e^{-i \theta} A\right)\right)\right\}
$$

where, for a complex number $z$ and a matrix $B, \operatorname{Re} z=(z+\bar{z}) / 2$ and $\operatorname{Re} B=$ $\left(B+B^{*}\right) / 2$ are their real parts, and, for an $n$-by- $n$ Hermitian matrix $C, \lambda_{1}(C) \geq$ $\cdots \geq \lambda_{n}(C)$ denote its eigenvalues in decreasing order. In particular, it follows that

$$
d(A)=\min \left\{i_{\geq 0}\left(\operatorname{Re}\left(e^{-i \theta} A\right)\right): \theta \in \mathbb{R}\right\}
$$

for any matrix $A$, where $i_{>0}\left(\operatorname{Re}\left(e^{-i \theta} A\right)\right)$ denotes the number of nonnegative eigenvalues of $\operatorname{Re}\left(e^{-i \theta} A\right)$ (cf. [4, Theorem 2.2]).

The purpose of this paper is to compute $d(A)$ when $A$ is the KMS matrix

$$
J_{n}(a)=\left[\begin{array}{ccccc}
0 & a & a^{2} & \cdots & a^{n-1} \\
& 0 & a & \ddots & \vdots \\
& & \ddots & \ddots & a^{2} \\
& & & \ddots & a \\
0 & & & & 0
\end{array}\right], \quad a \in \mathbb{C} \text { and } n \geq 1 \text {. }
$$

The study of the numerical range of $J_{n}(a)$ was started by Gaaya in [1, 2] and continued by the present authors in [5]. As a meeting ground of the classes of nilpotent, Toeplitz, nonnegative, $S_{n^{-}}$and $S_{n}^{-1}$-matrices, $J_{n}(a)$ has diverse and interesting properties concerning its numerical range. The present paper is a further exploration of such properties. In Section 2 below, we show that

$$
d\left(J_{n}(a)\right)= \begin{cases}n & \text { if } a=0 \\ k & \text { if } a \neq 0 \text { and } \cos \frac{k \pi}{n-1}<|a| \leq \cos \frac{(k-1) \pi}{n-1}, 1 \leq k \leq\left\lfloor\frac{n}{2}\right\rfloor \\ 1 & \text { if }|a|>1\end{cases}
$$


for any $n \geq 2$. This is proven via, in addition to the Li-Sze result, the congruence of $\operatorname{Re}\left(e^{-i \theta} J_{n}(a)\right)$ and the $n$-by- $n$ matrix

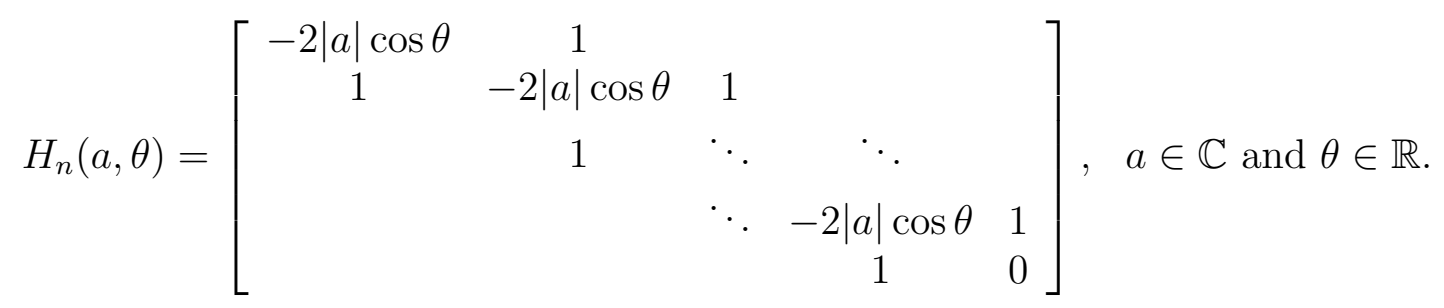

Here $H_{1}(a, \theta)$ is understood to be the 1-by-1 zero matrix. In the end of Section 2, we carry over the result for $J_{n}(a)$ to that for the classes of $S_{n^{-}}$and $S_{n}^{-1}$-matrices with one single eigenvalue.

In the following, we use diag $\left(a_{1}, \ldots, a_{n}\right)$ to denote the $n$-by- $n$ diagonal matrix with diagonals $a_{1}, \ldots, a_{n}$. For a subset $K$ of $\mathbb{C}^{n}, \bigvee K$ denotes the subspace of $\mathbb{C}^{n}$ generated by vectors in $K$. If $t$ is a real number, then $\lfloor t\rfloor$ (resp., $\lceil t\rceil$ ) denotes the largest (resp., smallest) integer less than (resp., greater than) or equal to $t$. Our reference for general properties of numerical ranges of matrices is [8, Chapter 1].

\section{MAin RESUlt}

The main result of this paper is the following theorem.

Theorem 2.1. For a in $\mathbb{C}$ and $n \geq 2$, we have

$$
\begin{aligned}
d\left(J_{n}(a)\right) & =i_{\geq 0}\left(\operatorname{Re} J_{n}(a)\right) \\
& = \begin{cases}n & \text { if } a=0, \\
k & \text { if } a \neq 0 \text { and } \cos \frac{k \pi}{n-1}<|a| \leq \cos \frac{(k-1) \pi}{n-1}, 1 \leq k \leq\left\lfloor\frac{n}{2}\right\rfloor, \\
1 & \text { if }|a|>1 .\end{cases}
\end{aligned}
$$

This will be proven after the next two lemmas, the first of which gives the congruence of $\operatorname{Re}\left(e^{-i \theta} J_{n}(a)\right)$ and $H_{n}(a, \theta)$ for any real $\theta$. Recall that two $n$-by- $n$ matrices $A$ and $B$ are congruent if $X A X^{*}=B$ for some invertible matrix $X$. By Sylvester's law of inertia [7, Theorem 4.5.8], two Hermitian matrices $A$ and $B$ are congruent if and only if they have the same numbers of positive, negative and zero eigenvalues. Thus, for congruent $A$ and $B$, we have $d(A)=d(B)$ by (1.1).

Lemma 2.2. If $a \neq 0$ in $\mathbb{C}$ and $n \geq 2$, then $\operatorname{Re}\left(e^{-i \theta} J_{n}(a)\right)$ is congruent to $H_{n}(a, \theta)$ for any real $\theta$.

Proof. Since $J_{n}(a)$ and $J_{n}(|a|)$ are unitarily similar by [5, Proposition 2.1 (a)], we may assume that $a>0$. Let $A=\operatorname{Re}\left(e^{-i \theta} J_{n}(a)\right), E_{j}=I_{j-1} \oplus\left[\begin{array}{cc}1 & -a \\ 0 & 1\end{array}\right] \oplus I_{n-j-1}$ for $1 \leq j \leq n-1$, and $E=E_{n-1} \cdots E_{2} E_{1}$. Then

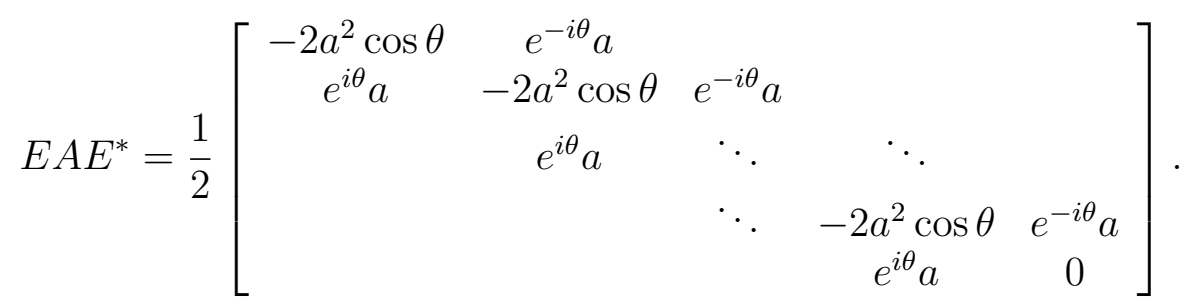


If $W=\sqrt{2 / a} \operatorname{diag}\left(1, e^{-i \theta}, e^{-2 i \theta}, \ldots, e^{-i(n-1) \theta}\right)$, then $W E A E^{*} W^{*}=H_{n}(a, \theta)$, which shows the congruence of $\operatorname{Re}\left(e^{-i \theta} J_{n}(a)\right)$ and $H_{n}(a, \theta)$.

For $n \geq 1$, let

$$
J_{n}=\left[\begin{array}{cccc}
0 & 1 & & \\
& 0 & \ddots & \\
& & \ddots & 1 \\
& & & 0
\end{array}\right]
$$

denote the $n$-by- $n$ Jordan block. It is known that the eigenvalues of $\operatorname{Re} J_{n}$ are $\cos (j \pi /(n+1)), 1 \leq j \leq n$ (cf. [6, p. 373]). The next lemma relates the two Hermitian matrices $H_{n}(a, \theta)$ and Re $J_{n-2}$.

Lemma 2.3. For any complex $a$, integer $n \geq 3$ and real $\theta$, the following hold:

(a) $\operatorname{det} H_{n}(a, \theta)=-2^{n-2} \operatorname{det}\left(\left(\operatorname{Re} J_{n-2}\right)-(|a| \cos \theta) I_{n-2}\right)$,

(b) 0 is an eigenvalue of $H_{n}(a, \theta)$ if and only if $|a| \cos \theta=\cos (j \pi /(n-1))$ for some $j, 1 \leq j \leq n-2$,

(c) $i_{\geq 0}\left(H_{n}(a, \theta)\right)=i_{\geq 0}\left(\left(\operatorname{Re} J_{n-2}\right)-(|a| \cos \theta) I_{n-2}\right)+1$, and

(d) $i_{\geq 0}\left(H_{n}\left(a, \theta_{1}\right)\right) \leq i_{\geq 0}\left(H_{n}\left(a, \theta_{2}\right)\right)$ for $0 \leq \theta_{1} \leq \theta_{2} \leq \pi$.

Proof. For convenience, let $A=H_{n}(a, \theta)$ and $B_{n}=2\left(\left(\operatorname{Re} J_{n}\right)-(|a| \cos \theta) I_{n}\right)$.

(a) To evaluate $\operatorname{det} A$, we expand it by minors on the last row of $A$ and then on the last column of the resulting $(n-1)$-by- $(n-1)$ submatrix to obtain

$$
\operatorname{det} A=-\operatorname{det} B_{n-2}=-2^{n-2} \operatorname{det}\left(\left(\operatorname{Re} J_{n-2}\right)-(|a| \cos \theta) I_{n-2}\right) .
$$

(b) This follows from (a) and the remark before the statement of this lemma.

(c) Note that $A$ is cyclic in the sense that there is a vector $x=\left[\begin{array}{lll}1 & 0 \ldots 0\end{array}\right]^{T}$ in $\mathbb{C}^{n}$ such that $x, A x, \ldots, A^{n-1} x$ generate $\mathbb{C}^{n}$. Hence $\mathbb{C}^{n}=\bigvee\left\{x,\left(A-\lambda I_{n}\right) x, \ldots,(A-\right.$ $\left.\left.\lambda I_{n}\right)^{n-1} x\right\}$ for any complex $\lambda$. If $\lambda$ is an eigenvalue of $A$, then the range of $A-\lambda I_{n}$ is not equal to $\mathbb{C}^{n}$ and thus, from above, $x$ is not in this range. In this case, we deduce that $\operatorname{rank}\left(A-\lambda I_{n}\right)=n-1$ or $\operatorname{dim} \operatorname{ker}\left(A-\lambda I_{n}\right)=1$. In particular, this shows that the eigenvalues of $A$ are all distinct. Let $\alpha_{1}>\alpha_{2}>\cdots>\alpha_{n}$ and $\beta_{1}>\beta_{2}>\cdots>\beta_{n-2}$ be the eigenvalues of $A$ and $B_{n-2}$, respectively. Since $B_{n-2}$ is a principal submatrix of $A$, the interlacing property for their eigenvalues $[7$, Theorem 4.3.8] yields that $\alpha_{j} \geq \beta_{j}$ for all $j, 1 \leq j \leq n-2$. If $\alpha_{j_{0}}=\beta_{j_{0}}$ for some $j_{0}$, then apply the interlacing property for $A, B_{n-1}$ and $B_{n-2}$ to infer that $\beta_{j_{0}}$ is also an eigenvalue of $B_{n-1}$. This is impossible since the eigenvalues of $B_{n-1}$ and $B_{n-2}$ are $2(\cos (j \pi / n)-|a| \cos \theta), 1 \leq j \leq n-1$, and $2(\cos (k \pi /(n-1))-|a| \cos \theta)$, $1 \leq k \leq n-2$, respectively, which are distinct from each other. Thus $\alpha_{j}>\beta_{j}$ for all $j, 1 \leq j \leq n-2$. Similarly, we have $\alpha_{j}<\beta_{j-2}$ for $3 \leq j \leq n$.

Let $k=i_{\geq 0}\left(B_{n-2}\right)$. If $|a| \cos \theta$ is an eigenvalue of $\operatorname{Re} J_{n-2}$, then 0 is an eigenvalue of $B_{n-2}$ and of $A$ by (b). From $\beta_{k-1}>0, \beta_{k}=0$ and $\beta_{k+1}<0$, we deduce that $\alpha_{k}>\beta_{k}=0, \alpha_{k+1}=0$ and $\alpha_{k+2}<\beta_{k}=0$. Therefore, $i_{\geq 0}(A)=k+1$ in this case. On the other hand, if $|a| \cos \theta$ is not an eigenvalue of $\operatorname{Re} J_{n-2}$, then the $\alpha_{j}$ 's and $\beta_{j}$ 's are all nonzero. From the preceding paragraph, we have $\alpha_{k}>\beta_{k}>0$ and $\alpha_{k+3}<\beta_{k+1}<0$. Since $\prod_{j=1}^{n} \alpha_{j}=-2^{n-2} \prod_{j=1}^{n-2} \beta_{j}$ by (a), we 
deduce that $\alpha_{k+1} \alpha_{k+2}<0$ and hence $\alpha_{k+1}>0>\alpha_{k+2}$. In this case, we again have $i_{\geq 0}(A)=k+1$.

(d) This is an easy consequence of (c).

We are now ready to prove Theorem 2.1.

Proof of Theorem 2.1. Assume that $a \neq 0$. If $n=2$, then a simple computation shows that the eigenvalues of $\operatorname{Re}\left(e^{-i \theta} J_{2}(a)\right)$ are $\pm|a| / 2$, and thus $d(A)=$ $i_{\geq 0}\left(\operatorname{Re} J_{2}(a)\right)=1$ by (1.1). For the remaining part of the proof, we assume that $n \geq 3$. Then a combination of (1.1) and Lemmas 2.2 and 2.3 (d) yields that

$$
\begin{aligned}
d\left(J_{n}(a)\right) & =\min \left\{i_{\geq 0}\left(\operatorname{Re}\left(e^{-i \theta} J_{n}(a)\right)\right): \theta \in \mathbb{R}\right\} \\
& =\min \left\{i_{\geq 0}\left(H_{n}(a, \theta)\right): \theta \in \mathbb{R}\right\} \\
& =i_{\geq 0}\left(H_{n}(a, 0)\right) \\
& =i_{\geq 0}\left(\operatorname{Re} J_{n}(a)\right) .
\end{aligned}
$$

Since $\left(\operatorname{Re} J_{n-2}\right)-|a| I_{n-2}$ has eigenvalues $\cos (j \pi /(n-1))-|a|, 1 \leq j \leq n-2$, if $\cos (k \pi /(n-1))<|a| \leq \cos ((k-1) \pi /(n-1))$ for some $k, 1 \leq k \leq\lfloor n / 2\rfloor$, then

$$
\begin{aligned}
d\left(J_{n}(a)\right) & =i_{\geq 0}\left(H_{n}(a, 0)\right)=i_{\geq 0}\left(\left(\operatorname{Re} J_{n-2}\right)-|a| I_{n-2}\right)+1 \\
& =(k-1)+1=k
\end{aligned}
$$

by Lemma 2.3 (c). Similarly, if $|a|>1$, then $d\left(J_{n}(a)\right)=1$.

The KMS matrices are closely related to those $S_{n^{-}}$and $S_{n}^{-1}$-matrices with one single eigenvalue. Recall that an $n$-by- $n$ matrix $A$ is said to be of class $S_{n}$ if it is a contraction, that is, $\|A\| \equiv \max _{\|x\|=1}\|A x\| \leq 1$, all its eigenvalues have moduli less than 1 , and $\operatorname{rank}\left(I_{n}-A^{*} A\right)=1$. It is of class $S_{n}^{-1}$ if all its eigenvalues have moduli greater than 1 and $\operatorname{rank}\left(I_{n}-A^{*} A\right)=1$. These two classes of matrices were first studied in [10] and [3], respectively. They are related to KMS matrices via affine functions: if $0<|a|<1$ (resp., $|a|>1)$, then $\left(\left(1-|a|^{2}\right) / a\right) J_{n}(a)-\bar{a} I_{n}$ is of class $S_{n}$ (resp., of class $S_{n}^{-1}$ ) with the single eigenvalue - $\bar{a}$ (cf. [5, Lemma 2.4]). Thus Theorem 2.1 may be transferred to one for $S_{n^{-}}$and $S_{n}^{-1}$-matrices.

Corollary 2.4. If $A$ is an $S_{n}$-matrix (resp., $S_{n}^{-1}$-matrix) with the single eigenvalue $\lambda$, then $d\left(A-\lambda I_{n}\right)=k$ for $\cos (k \pi /(n-1))<|\lambda| \leq \cos ((k-1) \pi /(n-1))$, $1 \leq k \leq\lfloor n / 2\rfloor$ (resp., $\left.d\left(A-\lambda I_{n}\right)=1\right)$.

We remark that, in the preceding corollary, $d\left(A-\lambda I_{n}\right)=1$ for $A$ an $S_{n}^{-1}$-matrix can also be proven by the result in [3]. Indeed, let $\lambda=|\lambda| e^{i \theta}$, where $0 \leq \theta<2 \pi$, and let $\lambda_{1} \geq \lambda_{2} \geq \cdots \geq \lambda_{n}$ be the eigenvalues of $\operatorname{Re}\left(e^{-i \theta} A\right)$. Since $\lambda$ is in $W(A)$, we have $\lambda_{1} \geq|\lambda|>1$. On the other hand, by [3, Lemma 2.9 (1)], we also have $\lambda_{2} \leq 1$. Thus the eigenvalues $\lambda_{j}-|\lambda|, 1 \leq j \leq n$, of $\operatorname{Re}\left(e^{-i \theta}\left(A-\lambda I_{n}\right)\right)$ are such that $\lambda_{1}-|\lambda| \geq 0$ and $\lambda_{2}-|\lambda|<\lambda_{2}-1 \leq 0$. Therefore, $d\left(A-\lambda I_{n}\right)=1$ by (1.1).

Acknowledgement. This research was partially supported by the National Science Council of the Republic of China under projects NSC-101-2115-M-008006 and NSC-101-2115-M-009-004 of the respective authors. The second author was also supported by the MOE-ATU. 


\section{REFERENCES}

1. H. Gaaya, On the numerical radius of the truncated adjoint shift, Extracta Math. 25 (2010), $165-182$.

2. H. Gaaya, A sharpened Schwarz-Pick operatorial inequality for nilpotent operators, arXiv: $1202.3962 \mathrm{v} 1$.

3. H.-L. Gau, Numerical ranges of reducible companion matrices, Linear Algebra Appl. 432 (2010), 1310-1321.

4. H.-L. Gau, K.-Z. Wang and P.Y. Wu, Zero-dilation index of a finite matrix, Linear Algebra Appl. arXiv: 1304.0296 (submitted).

5. H.-L. Gau and P.Y. Wu, Numerical Ranges of KMS matrices, Acta Sci. Math. (Szeged), arXiv: 1304.0295 (to appear).

6. U. Haagerup and P. de la Harpe, The numerical radius of a nilpotent operator on a Hilbert space, Proc. Amer. Math. Soc. 115 (1992), 371-379.

7. R.A. Horn and C.R. Johnson, Matrix Analysis, Cambridge University Press, Cambridge, 1985.

8. R.A. Horn and C.R. Johnson, Topics in Matrix Analysis, Cambridge University Press, Cambridge, 1991.

9. C.-K. Li and N.-S. Sze, Canonical forms, higher rank numerical ranges, totally isotropic subspaces, and matrix equations, Proc. Amer. Math. Soc. 136 (2008), 3013-3023.

10. D. Sarason, Generalized interpolation in $H^{\infty}$, Trans. Amer. Math. Soc. 127 (1967), 179203.

1 Department of Mathematics, National Central University, Chungli 32001, TAIWAN.

E-mail address: hlgau@math.ncu.edu.tw

2 Department of Applied Mathematics, National Chiao Tung University, Hsinchu 30010, TAIWAN.

E-mail address: pywu@math.nctu.edu.tw 